\title{
Antioxidant and Cytotoxic Activities of Clove Oil Nanoparticles and Evaluation of Its Size and Retention Efficiency
}

\author{
Clara Teixeira de Oliveira, Maria Inês Bruno Tavares \\ Institute of Macromolecules Professor Eloisa Mano, Universidade Federal do Rio de Janeiro, Rio de Janeiro, Brazil \\ Email: claratoliveira@gmail.com
}

How to cite this paper: de Oliveira, C.T. and Tavares, M.I.B. (2022) Antioxidant and Cytotoxic Activities of Clove Oil Nanoparticles and Evaluation of Its Size and Retention Efficiency. Materials Sciences and Applications, 13, 39-53.

https://doi.org/10.4236/msa.2022.131003

Received: November 24, 2021

Accepted: January 18, 2022

Published: January 21, 2022

Copyright $\odot 2022$ by author(s) and Scientific Research Publishing Inc. This work is licensed under the Creative Commons Attribution International License (CC BY 4.0).

http://creativecommons.org/licenses/by/4.0/

\begin{abstract}
A derivation of the nanoprecipitation technique without the presence of surfactants to reduce the nanoparticle size is herein proposed. The absence of surfactant in the nanoprecipitation technique allows capturing particles with a smaller diameter than nanoparticles containing surfactants, facilitating the migration of antioxidant nanoparticles in film packaging. Biodegradable PLA nanoparticles with clove oil were produced and characterized by dynamic light scattering, zeta potential, Fourier transform infrared spectroscopy, retention efficiency, cytotoxicity, and antioxidant activity. The particle sizes obtained were smaller than those commonly produced by nanoprecipitation, monodispersed and stable for 6 months. The antioxidant activity showed that the encapsulated form of clove oil had greater antioxidant activity than unencapsulated clove oil. The addition of PLA nanoparticles decreased the cytotoxic action of eugenol, the main antioxidant component of clove oil.
\end{abstract}

\section{Keywords}

Nanoprecipitation, PLA, Clove Oil, Antioxidant

\section{Introduction}

Changes in the profile of food consumption without synthetic additives modify the demand of food companies, stimulating the use of antimicrobial compounds in food formulations and packaging [1]. Although promised for the addition of active packaging, the use of inorganic nanoparticles still raises many doubts about the ecotoxicity and toxicity of these particles for the human organism in case of migration [2] [3].

In this context, a natural alternative for antimicrobial and antioxidant appli- 
cations are Essential Oils (EOs) that stand out due to their great potential and application in food packaging [4]. The gradual release of EOs during transport and storage can increase the shelf life and keep the food during shelf life [5]. Thus, the development of polymeric nanoparticles based on biodegradable and essential polymers may represent a more promising and safer perspective than inorganic particles in this application sector.

Essential oils are aromatic substances naturally produced by plants as secondary metabolites and have complex mixtures of terpenes, terpenoids, phenylpropanoids. They are also being considered as GRAS (generally recognized as safe) by the Food and Drug Administration (FDA) [6]. The composition is variable and plays an important role in determining their activity, being composed of phenolic groups more effective in terms of antioxidant and antimicrobial activities [7] [8] [9].

The antimicrobial action of essential oil-containing phenolic groups occurs with the release or migration on the food surface, rupture of this membrane and subsequent destruction of the cell. The phenolic compounds of the essentials can interact with the lipids and proteins present in the cell membranes of gram-positive and gram-negative bacteria produced in the death of the cell due to changes in their permeability, fluidity, inhibition of breathing and changes in ion transport. Among oils that have phenolic groups, oils from cloves, basil, cinnamon, peppermint, and oregano are the most consistent and effective against pathogenic microorganisms. This activity prolongs the shelf life of food and prevents the incidence of foodborne diseases caused by pathogenic microorganisms [10] [11] [12].

However, the direct incorporation of the essential oils into polymeric matrices can be a complicated process because these oils are susceptible to oxidation and degradation due to high temperature and shear6. A technique capable of mitigating these limitations is encapsulation, which can overcome active dosage limitations, increase its oxidation stability and high temperatures, and protect the alternative antimicrobial and antioxidant activity [13] [14].

When encapsulation produces particles at the nanoscale, the bioavailability and bio effectiveness of essential oils can be increased, because nanoparticles have larger surface areas than similar masses of larger-scale materials. This particle size also allows for the reduction of oil doses necessary to achieve antimicrobial and antioxidant activities [15] [16].

Among the methodologies used to produce nanoparticles, nanoprecipitation is a simple method proposed by FESSI and his collaborators (1989) and it is used for encapsulation of drugs, nutrients, or assets in nanoparticles, as it is an easy and reproducible method based on the interfacial polymer deposition after displacement of water-miscible semipolar solvent (organic phase) [17]. The size of the nanoparticles is dependent on the nature of the polymer and the higher the molecular mass and hydrophobicity of the polymer are, the greater the nanoparticles are produced. In addition, the absence of surfactant in the nanoprecipita- 
tion technique allows catching particles with a diameter smaller than nanoparticles containing surfactants [18]. The smallest particle diameters are particularly interesting in packaging applications because smaller particle diameters can facilitate the process of migration from the nanoparticle to the food, which is where in fact the antimicrobial and antioxidant actions are most important [19] [20] [21].

There are many polymers for the preparation of nanoparticles. The ones commonly used to encapsulate in nanoprecipitation are biodegradable polyesters, such as Polylactide (PLA), Polylactide-Coglycolide (PLGA) and Poly-Caprolactone (PCL). The choice of polymer, among other parameters, affects the properties of nanocapsules in terms of size, thermal stability, encapsulation efficiency and interaction with food [22]. Semicrystalline PLA, for example, is widely used to produce nanoparticles through nanoprecipitation, because this matrix has excellent biocompatibility, undergoes thermal degradation above $200^{\circ} \mathrm{C}$ and allows process ability with a wide range of techniques [23].

Due to the growing demand for researching in active packaging, this work aims to obtain PLA nanoparticles loaded with clove oil by nanoprecipitation and also do a detailed study to evaluate its potential application for the development of active packaging.

\section{Experimental}

\subsection{Nanoprecipitation}

The proposed method for preparing nanoparticles was developed through derivation of the nanoprecipitation method used by Oliveira et al. [18]. Thus, to obtain the PLA nanoparticles, two phases were obtained: an aqueous phase and an organic phase formed by a mixture of acetone from Sigma Aldrich $\geq 99.5 \%$ ) and THF from Vetec ${ }^{\mathrm{T} w}$, reagent grade, anhydrous, contains $100 \mathrm{ppm}$ BHT as inhibitor, 299.8\%) (1:1) solvents, containing the semi-crystalline PLA 4530D from NatureWorks LLC at $1.6 \mathrm{mg} / \mathrm{mL}$ and the clove oil from Bioessencia in mass contents $10 \%$, $20 \%$ and $30 \%$. The ratios 1:1 Acetone/THF and 1:2 of organic phase to aqueous were used. Initially, the solubilization of PLA in THF was carried out in a closed system, using light heating $\left(50^{\circ} \mathrm{C}\right)$ for $3 \mathrm{~h}$. Clove oil was solubilized in acetone in different proportions $(10 \%, 20 \%$ and $30 \%)$. The organic phase was then obtained from a mixture of THF containing PLA and Acetone containing clove oil in a 1:1 ratio of solvents. After this process, the systems containing 10\%, 20\% and 30\% of oil obtained remained under stirring for 72 hours in a hood to eliminate the organic solvents.

Aliquots of the colloidal suspensions were used for: characterization by Dynamic Light Scattering (DLS), Zeta Potential (ZP) and others were stored in a refrigerator at a temperature of $5^{\circ} \mathrm{C}$ for 90 days to verify the aging and coalescence of systems containing different proportions of oil. The remaining aliquots of the suspensions obtained were frozen in an ultra-freezer Liotop Model UFR30, Series 24,312 at $-90^{\circ} \mathrm{C}$ for 4 hours and then lyophilized (Liotop, Model L101) for 
96 hours $\left(-49^{\circ} \mathrm{C}\right.$ at $\left.72 \mathrm{mmHg}\right)$. The dry particles were characterized by dynamic light scattering, Zeta potential, Fourier Transform Infrared Spectroscopy, Retention Efficiency, Antioxidant Potential (DPPH) and Particle Cytotoxicity.

\subsection{Characterization}

Mean hydrodynamic diameters and polydispersity of the samples obtained were determined in the zetasizer Nano S90 model from Malvern Instruments, with 12 runs at $25^{\circ} \mathrm{C}$.

Zeta potential of each sample was measured using the Brookhaven Instruments Model NanoBrook Zeta PALS. For all analyzes 12 runs were performed and the dispersion and dilution conditions used were like those used in the DLS technique.

The FTIR analyzes were performed at $25^{\circ} \mathrm{C}$, in a Perkin Elmer Spectrum device version: 10.4.2 and Frontier Model FT-IR/FIR with DTGS detector in the Attenuated Total Reflection (ATR) function in the observation range from 400 to $4000 \mathrm{~cm}^{-1}$ using 64 scans.

Retention Efficiency (RE\%) of nanoparticles, was determined using the spectrophotometric method with a microplate spectrophometer from BioTek, model Winooski. The nanoparticles were separated from non-encapsulated free essential oil by centrifugation at $4000 \mathrm{rpm}$ for $10 \mathrm{~min}$. The supernatant was removed, and separated nanoparticles were redispersed and washed for a triple in distilled water by centrifuging at $4000 \mathrm{rpm}$ for another $10 \mathrm{~min}$. The pellet of each nanoparticle's formulation was extracted using dichloromethane as an extracting solvent, vortexed vigorously and centrifuged. The extracted oil was collected and quantified spectrophotometrically at $282 \mathrm{~nm}$, using a calibration curve.

DPPH evaluation method • (2,2-diphenyl-1-picrylhydrazyl) was used to estimate the antioxidant activity of the oil and the formulations. For this purpose, a solution of DPPH in methanol at a concentration of $6 \times 10^{-5} \mathrm{M}$ was obtained. A $5 \mathrm{ml}$ aliquot of this solution was placed in contact with the free oil and encapsulated as well as with samples of vitamin E and vitamin C (analysis standards). After 30 minutes of contact of the DDPH solution with the samples at an ambient temperature of $21^{\circ} \mathrm{C}$, the absorbance of the solutions was analyzed in a Visible Ultraviolet (UV-vis) spectrophotometer, Varian, model Cary 100 at a wavelength of $517 \mathrm{~nm}$.

The system cytotoxicity was evaluated in L929 lineage cells (provided from Rio de Janeiro Cell Bank-BCRJ) through neutral red dye analysis, following the methodology described by Narayanamurthy et al. (2019) [24] and ISO 10993-5 [25].

\section{Results and Discussion}

\subsection{Dynamic Light Scattering (DLS)}

Mean nanoparticle size of samples was varied between 60 and $87 \mathrm{~nm}$ (Figure 1) with a polydispersity index (PDI) 0.1 , indicating the homogeneity of nanoparticle population in the samples (Figure 2). The recovered systems further exhibited 

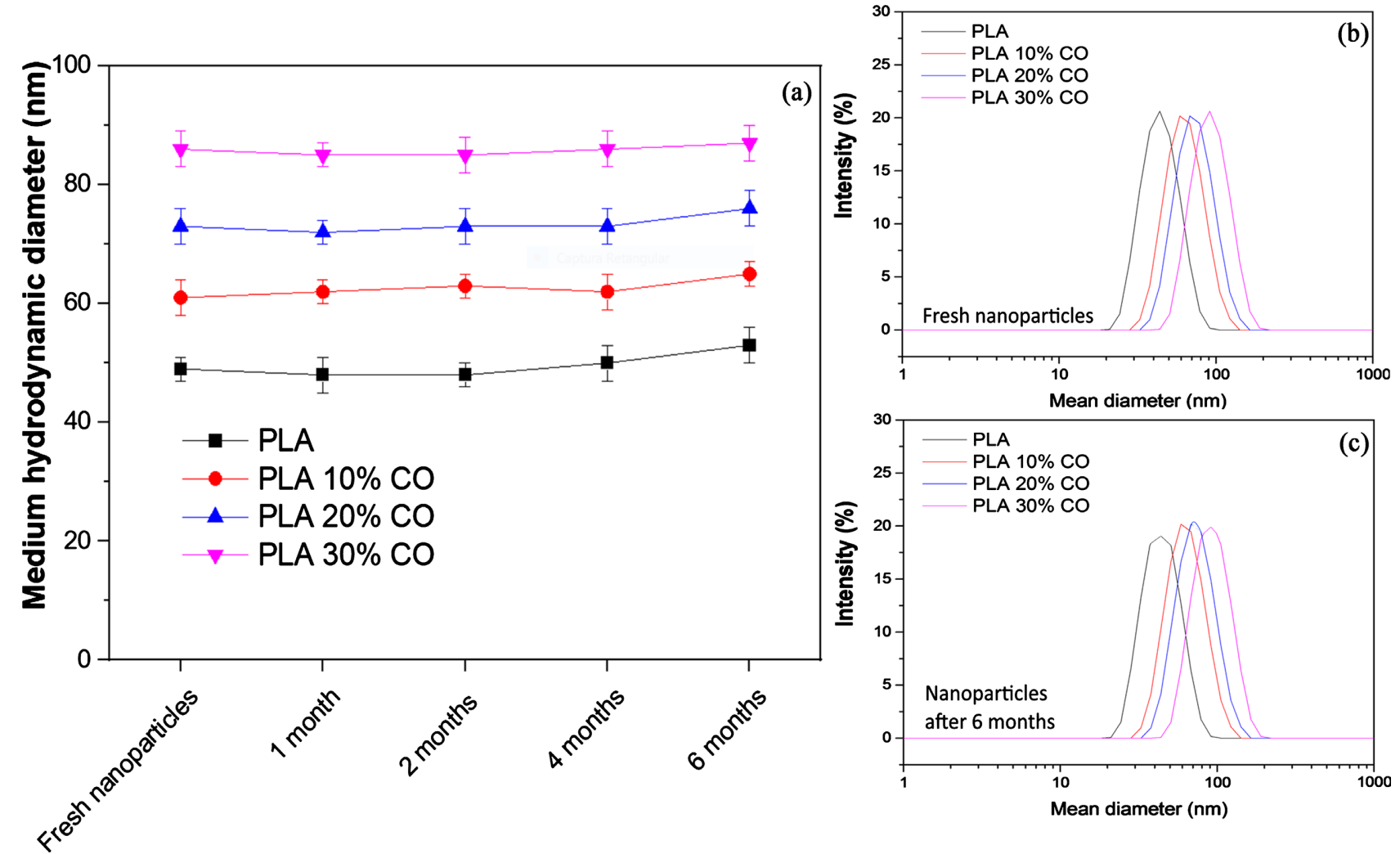

Figure 1. Medium hydrodynamic diameter of PLA/CO nanoparticles compared to pure PLA nanoparticle.

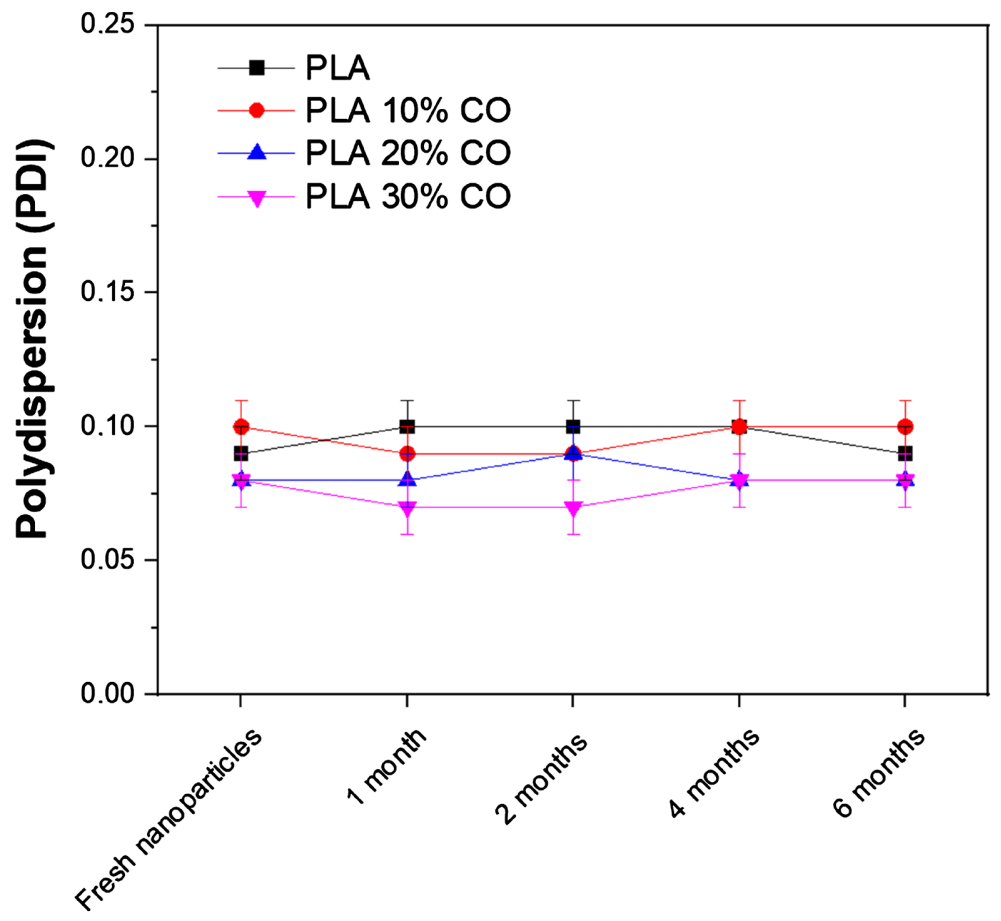

Figure 2. Polydispersion index of PLA/CO nanoparticles compared to pure PLA nanoparticle.

high colloidal stability amplified their size and stable PDI for up to 6 months. There are no size variations or changes in the polydisperse curves of the systems 
before (Figure 1(b)) and after 6 months of aging (Figure 1(c))

The formation of particles with low dispersion indexes is frequent in the nanoprecipitation technique. The physicochemical parameters for obtaining these particles, when well adjusted, lead to the formation of particles with narrow and monomodal size distributions. The results found are consistent with other works about this technique [26] [27] [28].

The particle sizes obtained are smaller than those commonly found for PLA particles obtained via nanoprecipitation, which may reflect the absence of surfactants in the adopted methodology [29]. The chemical and steric structure of the surfactants also influence the size and intensity of the particles, therefore, the absence of surfactants in this methodology allowed for smaller particle sizes [29] [30].

The medium hydrodynamic diameter increased proportionally to the increase in the concentration of added clove oil, related to the increase in the internal volume of the nanoparticle occupied by the oil. This increase in internal volume indicates the increase in the supply of $\mathrm{CO}$ within the particle as its concentration increases. This could be also attributed to the possible changes in the organic viscosity and physicochemical properties of solvent release into water [18].

Moreover, the time factor on nanoparticle size distribution is shown in Figure 3 for the same samples and did not show significant changes. The samples show particle stability for up to 6 months, indicating that the systems do not tend to undergo Ostwald coalescence and aging processes. The stability of these formulations, according to some authors, may be related to the polarity of this oil and its affinity with the materials chosen for the formulation of these systems [31] [32] [33]. In this perspective, systems containing compounds with more hydrophobic characteristics such as $\mathrm{CO}$ tend to have greater stability when encapsulated in hydrophobic matrices such as PLA [34].

\subsection{Zeta Potential (ZP)}

Table 1 shows PLA/CO zeta potential with $10 \%, 20 \%$ and $30 \%$ clove oil comparing to PLA. The load of pure PLA has a value of -22 . The negative value can be attributed to the presence of polyesters that are negatively ionized by the presence of carbonyl and carboxyl terminals. As the modulus is relatively high, these particles contribute to the elevation of systems that present colloidal stability,

Table 1. Zeta potential of nanoparticle systems.

\begin{tabular}{cc}
\hline Samples & PZ $(\mathrm{mV} \pm 1)$ \\
\hline PLA & $-22 \mathrm{a}^{\mathrm{b}}$ \\
PLA 10\%CO & $-22 \mathrm{a}$ \\
PLA 20\%CO & $-23 \mathrm{a}$ \\
PLA 30\%CO & $-23 \mathrm{a}$ \\
\hline
\end{tabular}

b. Means followed by different letters differ from each other $(\mathrm{p} \leq 0.05)$. 
corroborating the findings by the stability data evaluated by DLS. There is no difference of charge when the clove oil is inside the PLA, indicating physical stability of the particles in the nano dispersion.

The PZ values found for these systems are consistent with other studies on non-surfactant coated PLA particles that exhibit a PZ value between -20 and -25 $\mathrm{mV}$ [35] [36]. These systems tend to achieve high stability because of this surface potential that inhibits aggregation of particles by collision processes for their Brownian motion. The ZP values can serve as indicative of the repulsion or particle aggregation behavior when in a colloidal suspension. Particles with high values in module, both negative and positive tend to indicate systems with greater electrostatic stability. According to some authors, it is desirable zeta potential equal to or higher than $30 \mathrm{mV}$ to stabilize the module spherical particles [37] [38].

\subsection{Fourier Transform Infrared Spectroscopy (FTIR)}

FTIR was used to evaluate the incorporation of clove essential oil in polymeric matrix. Figure 3 shows the FTIR peaks of PLA (matrix), clove oil and clove oil nanoparticles. The FTIR spectra profile presents the profiles for the PLA matrix and for clove oil [39]. FTIR analysis showed the incorporation of clove oil in PLA nanoparticles. It was evidenced with the presence of chemical bonds specific to eugenol, a major compound of clove oil. Furthermore, no significant changes are observed regarding the shape, intensity and positioning of the PLA bands. This result indicates the non-occurrence of chemical bonding between the matrix and the encapsulated oil in encapsulation of natural compounds in polyester matrices [40].

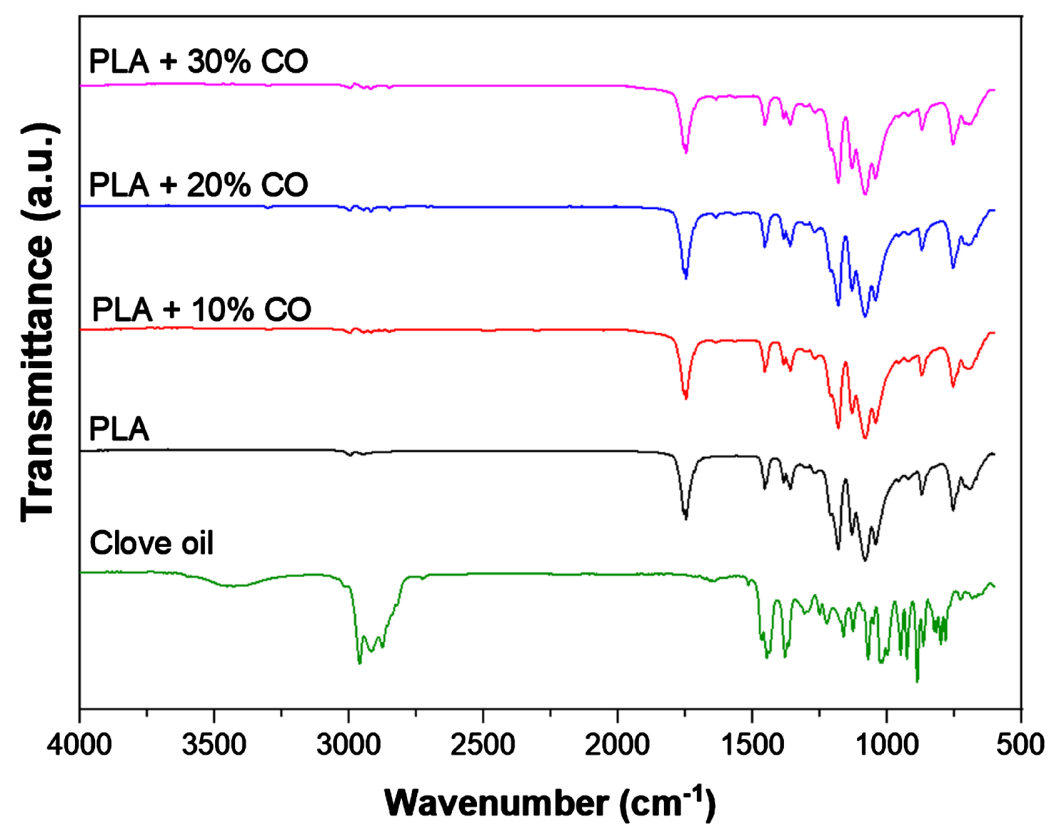

Figure 3. Infrared spectroscopy analysis of PLA/CO systems compared to pure clove oil and pure PLA to verify the insertion of oil into the nanoparticle. 
Figure 3 enlists the peaks observed due to bond vibration of functional groups and basic aromatic structure. The characteristic absorbance peaks of phenolic $\mathrm{O}-\mathrm{H}$ and C-H stretching in aromatic ring at $3492 \mathrm{~cm}^{-1}$ and $2960 \mathrm{~cm}^{-1}$, respectively were observed that were attributed to $\mathrm{O}-\mathrm{H}$ group and aromatic structure of eugenol. The $\mathrm{C}-\mathrm{H}$-stretching peak at $3003 \mathrm{~cm}^{-1}$ was observed due to allyl group (C-H attached to olefin) in eugenol.

The PLA has the typical bands in the following regions: carbonyl groups, $\mathrm{C}=\mathrm{O}$, at $1755 \mathrm{~cm}^{-1}$ and bending of $-\mathrm{CH}_{3}$, antisymmetric at $1454 \mathrm{~cm}^{-1}$ and symmetric at $1361 \mathrm{~cm}^{-1}$. Likewise, the stretching vibrations of C-O groups are at $1200 \mathrm{~cm}^{-1}$ and at $1050 \mathrm{~cm}^{-1}$. It is also possible to identify at 920 and $956 \mathrm{~cm}^{-1}$ those corresponding to the main chain vibrations (rocking of $\mathrm{CH} 3$ ), and at 869 and $755 \mathrm{~cm}^{-1}$, which can be assigned to the amorphous and crystalline phases of PLA, respectively. Polylactic acid encapsulating clove oil exhibits three small peaks at wavenumbers 1767 and $1732 \mathrm{~cm}^{-1}$. These peaks may be due to the $-\mathrm{C}=\mathrm{O}$ stretch in acetate attached to aromatic ring indicating the presence of clove oil in the nanoparticle sample [41] [42].

\subsection{Retention Efficiency (RE)}

Table 2 contains the retention efficiency of clove oil in PLA. Values were obtained between $83 \%$ and $86 \%$ for the obtained systems. The increase in oil concentration leads to a reduction in the retention efficiency, which may indicate a saturation of these systems.

The incorporation of the active nanoparticles obtained by this technique greatly varies over the work available in the literature. These variations are mainly due to the polymer matrix and active pair used in each case [43] [44]. The values found are shown much higher than in Casein performs encapsulating eugenol from clove oil, Which Reached 63.86\% encapsulation efficiency. Retentions above $80 \%$ are good retentions for the technique, possibly suggesting the greater the more hydrophobic characteristic of the oil, which favors its presence in the PLA phase over the aqueous phase, favoring greater encapsulations [33] [34] [45].

\subsection{Antioxidant Activity (DPPH)}

Antioxidant activity results show the EC50 of the tested formulations. In this test, the active content necessary to stabilize $50 \%$ of the free radicals generated by the reagent used is measured. Thus, it can be established that the lower the required sample content, the greater its antioxidant activity. Vitamin C and E samples

Table 2. Oil retention efficiency in nanoparticles.

\begin{tabular}{cc}
\hline Samples & RE (\%) \\
\hline PLA 10\% CO & $86 \pm 2$ \\
PLA 20\% CO & $87 \pm 3$ \\
PLA 30\% CO & $83 \pm 2$ \\
\hline
\end{tabular}


were used as known standards for analysis. Based on this, it can be established that clove oil in its free state or after encapsulation has high antioxidant activity, which is statistically like the antioxidant potential of vitamin E.

Nanoencapsulation is known to increase the antioxidant potential of natural molecules. Therefore, free radical scavenging activity was evaluated by measuring the scavenging activity of the clove oil nanoparticle (PLA/CO) on the DPPH radical. DPPH showed lower EC50 values for PLA/CO nanoparticles compared to pure clove oil, meaning protection from the oxidation of the oil by the nanoparticle [46] [47].

The free and encapsulated clove oil samples were also evaluated after exposure to heat, to determine the protective capacity of the active by the PLA matrix. After exposing the samples to heat, a decrease in the antioxidant activity of pure oil can be observed. The EC50 values of the nanoparticle were lower than those of clove oil, indicating thermal protection of the oil by PLA. PLA/CO reduced DPPH radical formation in a dose-dependent manner, with $30 \%$ CON clove oil being more effective with an EC50 of $34 \mu \mathrm{g} / \mathrm{mL}$ after exposure to $120^{\circ} \mathrm{C}$, when compared to pure clove oil, which was $43 \mu \mathrm{g} / \mathrm{mL}$, indicating thermal protection of the oil through nanoencapsulation. As shown in Figure 4, the results showed that the encapsulated form of clove oil had much greater antioxidant activity than unencapsulated clove oil.

\subsection{Particle's Cytotoxicity}

The results of cell viability of the L929 fibroblast lineage cells after 24 hours contact

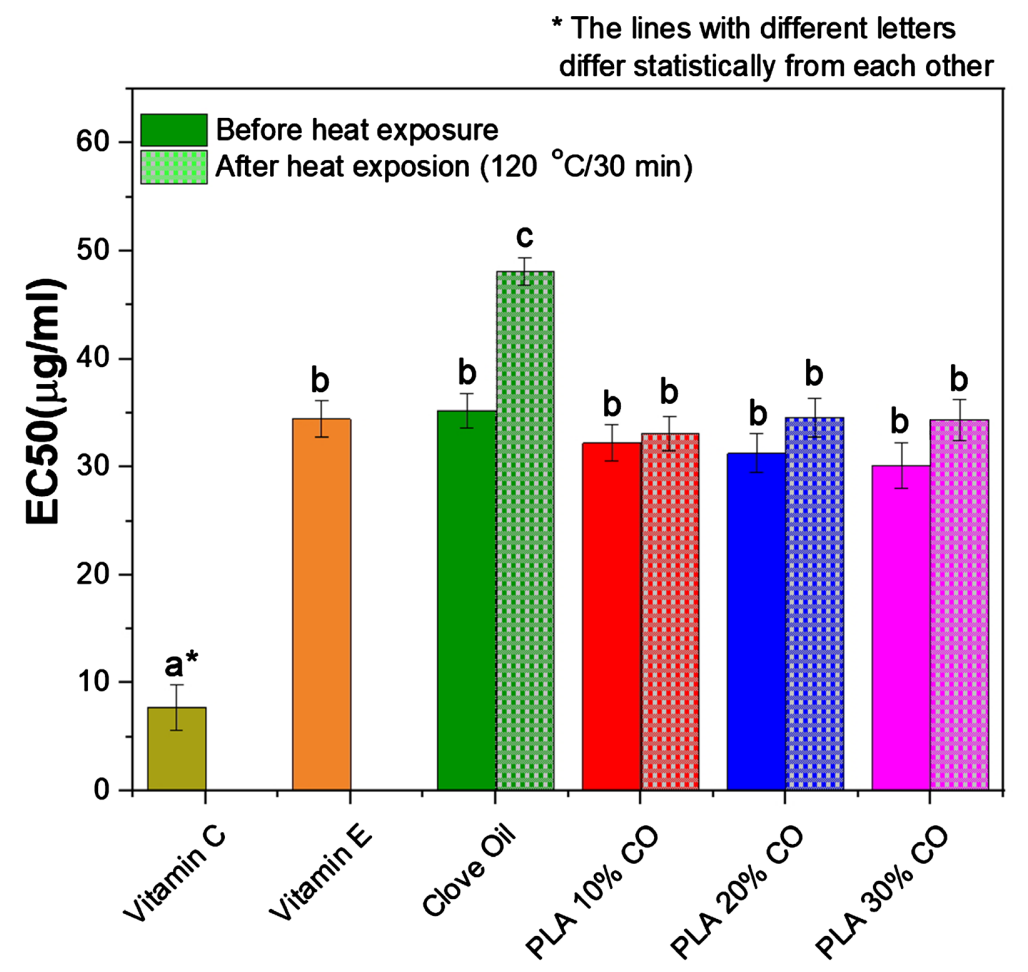

Figure 4. Antioxidant activity results of PLA/CO nanoparticles compared to clove oil. 


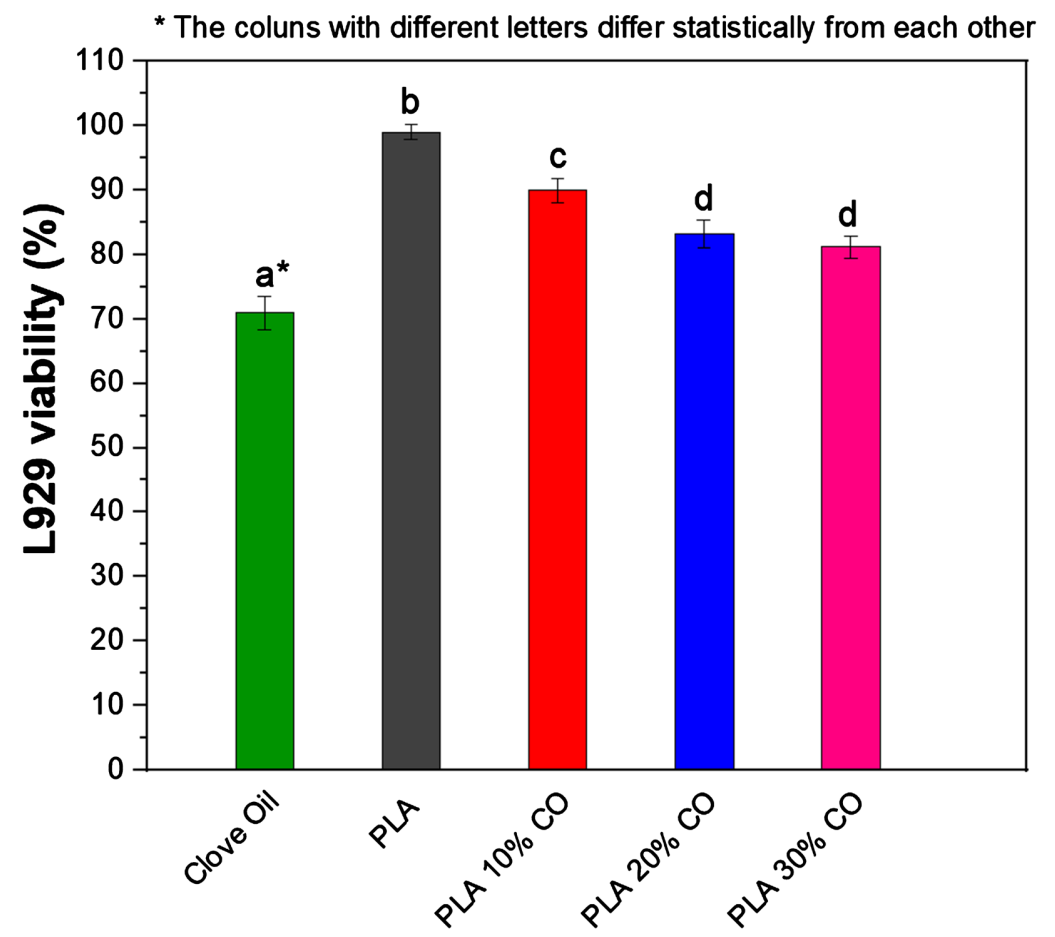

Figure 5. Cytotoxicity results of PLA/CO nanoparticles compared to clove oil and pure PLA.

of the cells with the formulations show different performance for those evaluated (Figure 5). Pure PLA nanoparticles do not generate any damage to the evaluated strain exhibiting viability close to $100 \%$. Nanoparticles containing clove oil generate reductions in cell viability, being more intense in formulations of 20 and $30 \%$ of $\mathrm{CO}$. Therefore, this effect is dependent on the oil content in the formulations. Finally, the pure oil generated greater viability reductions, generating a final viability around $72 \%$, which shows its slightly cytotoxic character.

It should be noted that even with reductions in cell viability, encapsulated samples can be considered non-cytotoxic, since, according to the ISO 10993-5: 200925, the evaluation of materials in contact with cell lines in vitro can only be considered cytotoxic when they lead to viability below $75 \%$.

The addition of PLA nanoparticles mitigated the cytotoxic action of eugenol, indicating the protection of the active by controlling the release of the active in the environment. Nanoparticles containing $20 \%$ and $30 \%$ clove essential oil have very close cytotoxicity curves, which may explain the impact of retention efficiency in cytotoxity [48] [49] [50]. The pure PLA nanoparticle results are consistent with the literature that emphasizes the high biocompatibility of PLA and its derivatives in different cell lines [51] [52] [53].

\section{Conclusion}

Nanoprecipitation has high encapsulation efficiency and can improve the stability of clove oil to oxidation, humidity, and high temperatures and, therefore, improve its efficiency. The absence of surfactants allows the reduction of the size ob- 
tained from the nanoparticles, favoring their migration to the food and consequently they have antimicrobial and antioxidative action. The use of natural antimicrobial agents, such as clove oil, applied with new nanotechnological techniques, allowing the successful development of new antimicrobial packaging for food preservation [13] [54].

\section{Conflicts of Interest}

The authors declare no conflicts of interest regarding the publication of this paper.

\section{References}

[1] Bahrami, A., Delshadi, R., Assadpour, E., Jafari, S.M. and Williams, L. (2020) Antimicrobial-Loaded Nanocarriers for Food Packaging Applications. Advances in Colloid and Interface Science, 278, Article ID: 102140. https://doi.org/10.1016/j.cis.2020.102140

[2] Biswas, J.K., Rai, M., Ingle, A.P., Mondal, M. and Biswas, S. (2018) Nano-Bio Interactions and Ecotoxicity in Aquatic Environment: Plenty of Room at the Bottom but Tyranny at the Top! In: Nanomaterials. Ecotoxicity, Safety, and Public Perception, Springer International Publishing, Berlin, 19-36. https://doi.org/10.1007/978-3-030-05144-0 2

[3] Brandelli, A. (2020) The Interaction of Nanostructured Antimicrobials with Biological Systems: Cellular Uptake, Trafficking and Potential Toxicity. Food Science and Human Wellness, 9, 8-20. https://doi.org/10.1016/j.fshw.2019.12.003

[4] Sutha, M., Selvaraj, R., Kulkarni, V.V., Chandirasekaran, V., Edwin, S.C. and Malmarugan, S. (2018) Effect of Rosemary Essential Oil on the Quality Characteristics of Chicken Nuggets. International Journal of Current Microbiology and Applied Sciences, 7, 4686-4693. https://doi.org/10.20546/ijcmas.2018.708.492

[5] Ju, J., Chen, X., Xie, Y., Yu, H., Guo, Y., Cheng, Y., Qian, H. and Yao, W. (2019) Application of Essential Oil as a Sustained Release Preparation in Food Packaging. Trends in Food Science \& Technology, 92, 22-32. https://doi.org/10.1016/j.tifs.2019.08.005

[6] Ribeiro-Santos, R., Andrade, M. and Sanches-Silva, A. (2017) Application of Encapsulated Essential Oils as Antimicrobial Agents in Food Packaging. Current Opinion in Food Science, 14, 78-84. https://doi.org/10.1016/j.cofs.2017.01.012

[7] Bhargava, K., Conti, D.S., da Rocha, S.R.P. and Zhang, Y. (2015) Application of an Oregano Oil Nanoemulsion to the Control of Foodborne Bacteria on Fresh Lettuce. Food Microbiology, 47, 69-73. https://doi.org/10.1016/j.fm.2014.11.007

[8] He, X. and Hwang, H.-M. (2016) Nanotechnology in Food Science: Functionality, Applicability, and Safety Assessment. Journal of Food and Drug Analysis, 24, 671-681. https://doi.org/10.1016/j.jfda.2016.06.001

[9] Becerril, R., Nerín, C. and Silva, F. (2020) Encapsulation Systems for Antimicrobial Food Packaging Components: An Update. Molecules, 25, 1134.

https://doi.org/10.3390/molecules25051134

[10] Hossain, M.A., Al-Hashmi, R.A., Weli, A.M., Al-Riyami, Q. and Al-Sabahib, J.N. (2012) Constituents of the Essential Oil from Different Brands of Syzigium caryophyllatum L by Gas Chromatography-Mass Spectrometry. Asian Pacific Journal of Tropical Biomedicine, 2, S1446-S1449. https://doi.org/10.1016/S2221-1691(12)60435-3

[11] Sadaka, F., Nguimjeu, C., Brachais, C.-H., Vroman, I., Tighzert, L. and Couvercelle, 
J.-P. (2013) WITHDRAWN: Review on Antimicrobial Packaging Containing Essential Oils and Their Active Biomolecules. Innovative Food Science \& Emerging Technologies, 20, 350. https://doi.org/10.1016/j.ifset.2013.01.004

[12] Neela, E. and Sandhu, H.K. (2019) Food Packaging Development: Recent Perspective. Journal of Thin Films, Coating Science Technology and Application, 6, 2-17. http://engineeringjournals.stmjournals.in/index.php/JoTCSTA/article/view/3511

[13] Shamaei, S., Seiiedlou, S.S., Aghbashlo, M., Tsotsas, E. and Kharaghani, A. (2017) Microencapsulation of Walnut Oil by Spray Drying: Effects of Wall Material and Drying Conditions on Physicochemical Properties of Microcapsules. Innovative Food Science \& Emerging Technologies, 39, 101-112. https://doi.org/10.1016/j.ifset.2016.11.011

[14] Asbahani, A.E., Miladi, K., Badri, W., Sala, M., Addi, E.H.A., Casabianca, H., Mousadik, A.E., Hartmann, D., Jilale, A., Renaud, F.N.R. and Elaissari, A. (2015) Essential Oils: From Extraction to Encapsulation. International Journal of Pharmaceutics, 483, 220-243. https://doi.org/10.1016/j.ijpharm.2014.12.069

[15] Hossain, F., Follett, P., Salmieri, S., Vu, K.D., Fraschini, C. and Lacroix, M. (2019) Antifungal Activities of Combined Treatments of Irradiation and Essential Oils (EOs) Encapsulated Chitosan Nanocomposite Films in In Vitro and In Situ Conditions. International Journal of Food Microbiology, 295, 33-40.

https://doi.org/10.1016/j.ijfoodmicro.2019.02.009

[16] Tiwari, S., Singh, B.K. and Dubey, N.K. (2020) Encapsulation of Essential Oils-A Booster to Enhance their Bio-Efficacy as Botanical Preservatives. Journal of Scientific Research, 64, 175-178. https://doi.org/10.37398/ISR.2020.640125

[17] Fessi, H., Puisieux, F., Devissaguet, J.P., Ammoury, N. and Benita, S. (1989) Nanocapsule Formation by Interfacial Polymer Deposition Following Solvent Displacement. International Journal of Pharmaceutics, 55, R1-R4. https://doi.org/10.1016/0378-5173(89)90281-0

[18] de Oliveira, A.M., Jäger, E., Jäger, A., Stepánek, P. and Giacomelli, F.C. (2013) Physicochemical Aspects behind the Size of Biodegradable Polymeric Nanoparticles: A Step Forward. Colloids and Surfaces A: Physicochemical and Engineering Aspects, 436, 1092-1102. https://doi.org/10.1016/j.colsurfa.2013.08.056

[19] Cushen, M., Kerry, J., Morris, M., Cruz-Romero, M. and Cummins, E. (2012) Nanotechnologies in the Food Industry-Recent Developments, Risks and Regulation. Trends in Food Science \& Technology, 24, 30-46. https://doi.org/10.1016/j.tifs.2011.10.006

[20] Honarvar, Z., Hadian, Z. and Mashayekh, M. (2016) Nanocomposites in Food Packaging Applications and Their Risk Assessment for Health. Electronic Physician, 8, 2531-2538. https://doi.org/10.19082/2531

[21] Enescu, D., Cerqueira, M.A., Fucinos, P. and Pastrana, L.M. (2019) Recent Advances and Challenges on Applications of Nanotechnology in Food Packaging. A Literature Review. Food and Chemical Toxicology, 134, Article ID: 110814. https://doi.org/10.1016/j.fct.2019.110814

[22] Rivas, C.J.M., Tarhini, M., Badri, W., Miladi, K., Greige-Gerges, H., Nazari, Q.A., Rodríguez, S.A.G., Román, R.Á., Fessi, H. and Elaissari, A. (2017) Nanoprecipitation Process: From Encapsulation to Drug Delivery. International Journal of Pharmaceutics, 532, 66-81. https://doi.org/10.1016/j.ijpharm.2017.08.064

[23] Casalini, T., Rossi, F., Castrovinci, A. and Perale, G. (2019) A Perspective on Polylactic Acid-Based Polymers Use for Nanoparticles Synthesis and Applications. Frontiers in Bioengineering and Biotechnology, 7, 259. https://doi.org/10.3389/fbioe.2019.00259 
[24] Narayanamurthy, V., Samsuri, F., Khan, A.Y.F., Hamzah, H.A., Baharom, M.B., Kumary, T.V., Kumar, P.R.A. and Raj, D.K. (2019) Direct Cell Imprint Lithography in Superconductive Carbon Black Polymer Composites: Process Optimization, Characterization and In Vitro Toxicity Analysis. Bioinspiration \& Biomimetics, 15, Article ID: 016002. https://doi.org/10.1088/1748-3190/ab1243

[25] ISO 10993-5:2009 (2009) Biological Evaluation of Medical Devices-Part 5: Tests for in Vitro Cytotoxicity; German Version EN ISO 10993-5:2009. https://www.iso.org/standard/36406.html

[26] Miladi, K., Sfar, S., Fessi, H. and Elaissari, A. (2016) Nanoprecipitation Process: From Particle Preparation to In Vivo Applications. In: Polymer Nanoparticles for Nanomedicines, Springer International Publishing, Berlin, 17-53.

https://doi.org/10.1007/978-3-319-41421-8 2

[27] Tavares, M.R., Menezes, L.R.D., Filho, J.C.D., Cabral, L.M. and Tavares, M.I.B. (2017) Surface-Coated Polycaprolactone Nanoparticles with Pharmaceutical Application: Structural and Molecular Mobility Evaluation by TD-NMR. Polymer Testing, 60, 39-48. https://doi.org/10.1016/j.polymertesting.2017.01.032

[28] da Rocha, L.V.M., Merat, L.C., de Menezes, L.R., Finotelli, P.V., da Silva, P.S.R.C. and Tavares, M.I.B. (2019) Extract of Curcuminoids Loaded on Polycaprolactone and Pluronic Nanoparticles: Chemical and Structural Properties. Applied Nanoscience, 10, 1141-1156. https://doi.org/10.1007/s13204-019-01197-w

[29] Hong, J.S., Srivastava, D. and Lee, I. (2018) Fabrication of Poly(lactic acid) Nanoand Microparticles Using a Nanomixer via Nanoprecipitation or Emulsion Diffusion. Journal of Applied Polymer Science, 135, 46199. https://doi.org/10.1002/app.46199

[30] Lindner, M., Bäumler, M. and Stäbler, A. (2018) Inter-Correlation among the Hydrophilic-Lipophilic Balance, Surfactant System, Viscosity, Particle Size, and Stability of Candelilla Wax-Based Dispersions. Coatings, 8, 469.

https://doi.org/10.3390/coatings8120469

[31] Wan, K.Y., Wong, K.W., Chow, A.H.L. and Chow, S.F. (2018) Impact of Molecular Rearrangement of Amphiphilic Stabilizers on Physical Stability of Itraconazole Nanoparticles Prepared by Flash Nanoprecipitation. International Journal of Pharmaceutics, 542, 221-231. https://doi.org/10.1016/j.ijpharm.2018.03.006

[32] Chow, S.F., Wan, K.Y., Cheng, K.K., Wong, K.W., Sun, C.C., Baum, L. and Chow, A.H.L. (2015) Development of Highly Stabilized Curcumin Nanoparticles by Flash Nanoprecipitation and Lyophilization. European Journal of Pharmaceutics and Biopharmaceutics, 94, 436-449. https://doi.org/10.1016/j.ejpb.2015.06.022

[33] Liu, Y., Yang, G., Zou, D., Hui, Y., Nigam, K., Middelberg, A.P.J. and Zhao, C.-X. (2019) Formulation of Nanoparticles Using Mixing-Induced Nanoprecipitation for Drug Delivery. Industrial \& Engineering Chemistry Research, 59, 4134-4149. https://doi.org/10.1021/acs.iecr.9b04747

[34] Zhu, Z. (2014) Flash Nanoprecipitation: Prediction and Enhancement of Particle Stability via Drug Structure. Molecular Pharmaceutics, 11, 776-786. https://doi.org/10.1021/mp500025e

[35] Mainardes, R.M., Khalil, N.M. and Gremião, M.P.D. (2010) Intranasal Delivery of Zidovudine by PLA and PLA-PEG Blend Nanoparticles. International Journal of Pharmaceutics, 395, 266-271. https://doi.org/10.1016/j.ijpharm.2010.05.020

[36] Kumari, P. and Dang, S. (2020) Development and In Vitro Characterization of Diazepam Loaded PLA Nanoparticles. Materials Today. Proceedings, 28, 246-250. https://doi.org/10.1016/j.matpr.2020.01.599 
[37] Somvanshi, S.B., Patade, S.R., Andhare, D.D., Jadhav, S.A., Khedkar, M.V., Kharat, P.B., Khirade, P.P. and Jadhav, K.M. (2020) Hyperthermic Evaluation of Oleic Acid Coated Nano-Spinel Magnesium Ferrite: Enhancement via Hydrophobic-to-Hydrophilic Surface Transformation. Journal of Alloys and Compounds, 835, Article ID: 155422. https://doi.org/10.1016/j.jallcom.2020.155422

[38] Kaasalainen, M., Mäkilä, E., Riikonen, J., Kovalainen, M., Järvinen, K., Herzig, K.-H., Lehto, V.-P. and Salonen, J. (2012) Effect of Isotonic Solutions and Peptide Adsorption on Zeta Potential of Porous Silicon Nanoparticle Drug Delivery Formulations. International Journal of Pharmaceutics, 431, 230-236.

https://doi.org/10.1016/j.ijpharm.2012.04.059

[39] Nagaraju, P.G., Sengupta, P., Chicgovinda, P.P. and Rao, P.J. (2021) Nanoencapsulation of Clove Oil and Study of Physicochemical Properties, Cytotoxic, Hemolytic, and Antioxidant Activities. Journal of Food Process Engineering, 44, e13645. https://doi.org/10.1111/jfpe.13645

[40] Ferreira, I.J., de Menezes, L.R. and Tavares, M.I.B. (2021) Morphological and Structural Evaluation of Nanoparticles Loaded with Tea Tree Oil for the Therapeutic Treatment of HPV. Polymer Bulletin, 70. https://doi.org/10.1007/s00289-021-03780-0

[41] Singh, G., Maurya, S., Catalan, C. and de Lampasona, M.P. (2004) Chemical Constituents, Antifungal and Antioxidative Effects of Ajwain Essential Oil and Its Acetone Extract. Journal of Agricultural and Food Chemistry, 52, 3292-3296. https://doi.org/10.1021/jf035211c

[42] Upadhyaya, S. (2014) Isolation and Characterization of a Bioactive Phenylpropanoid from Ocimum sanctum L. Leaves through Chromatographic and Spectroscopic Methods. American Chemical Science Journal, 4, 286-297. https://doi.org/10.9734/ACSJ/2014/6609

[43] Dehkharghani, R.A., Doust, M.Z., Kheiri, M.T. and Shahi, H.H. (2018) Impacts of Chemical Variables on the Encapsulated Corticoids in Poly-E-caprolactone Nanoparticles and Statistical Biological Analysis. Russian Journal of Applied Chemistry, 91, 1165-1171. https://doi.org/10.1134/S1070427218070157

[44] Diyanat, M. and Saeidian, H. (2019) The Metribuzin Herbicide in Polycaprolactone Nanocapsules Shows Less Plant Chromosome Aberration than Non-Encapsulated Metribuzin. Environmental Chemistry Letters, 17, 1881-1888. https://doi.org/10.1007/s10311-019-00912-x

[45] Wijayanto, A., Putri, Y.R.P., Hermansyah, H. and Sahlan, M. (2017) Encapsulation of Eugenol from Clove Oil Using Casein Micelle for Solid Preparation. AIP Conference Proceedings, 1817, Article ID: 030012. https://doi.org/10.1063/1.4976781

[46] dos Santos, P.D.F., Francisco, C.R.L., Coqueiro, A., Leimann, F.V., Pinela, J., Calhelha, R.C., Ineu, R.P., Ferreira, I.C.F.R., Bona, E. and Gonçalves, O.H. (2019) The Nanoencapsulation of Curcuminoids Extracted from Curcuma longa L. and an Evaluation of Their Cytotoxic, Enzymatic, Antioxidant and Anti-Inflammatory Activities. Food \& Function, 10, 573-582. https://doi.org/10.1039/C8FO02431F

[47] Huang, X., Liu, Y., Zou, Y., Liang, X., Peng, Y., McClements, D.J. and Hu, K. (2019) Encapsulation of Resveratrol in Zein/Pectin Core-Shell Nanoparticles: Stability, Bioaccessibility, and Antioxidant Capacity after Simulated Gastrointestinal Digestion. Food Hydrocolloids, 93, 261-269. https://doi.org/10.1016/j.foodhyd.2019.02.039

[48] Hume, W.R. (1984) Basic Biological Sciences Effect of Eugenol on Respiration and Division in Human Pulp, Mouse Fibroblasts, and Liver Cells In Vitro. Journal of Dental Research, 63, 1262-1265. https://doi.org/10.1177/00220345840630110101

[49] Ho, Y.C., Huang, F.M. and Chang, Y.C. (2006) Mechanisms of Cytotoxicity of Eu- 
genol in Human Osteoblastic Cells In Vitro. International Endodontic Journal, 39, 389-393. https://doi.org/10.1111/j.1365-2591.2006.01091.x

[50] Tai, K., Huang, F. and Chang, Y. (2001) Cytotoxic Evaluation of Root Canal Filling Materials on Primary Human Oral Fibroblast Cultures and a Permanent Hamster Cell Line. Journal of Endodontics, 27, 571-573. https://doi.org/10.1097/00004770-200109000-00004

[51] Tsou, C.-H., Yao, W.-H., Lu, Y.-C., Tsou, C.-Y., Wu, C.-S., Chen, J., Wang, R., Su, C., Hung, W.-S., De Guzman, M. and Suen, M.-C. (2017) Antibacterial Property and Cytotoxicity of a Poly(Lactic Acid)/Nanosilver-Doped Multiwall Carbon Nanotube Nanocomposite. Polymers, 9, 100. https://doi.org/10.3390/polym9030100

[52] Mania, S., Partyka, K., Pilch, J., Augustin, E., Cieślik, M., Ryl, J., Jinn, J.-R., Wang, Y.-J., Michałowska, A. and Tylingo, R. (2019) Obtaining and Characterization of the PLA/Chitosan Foams with Antimicrobial Properties Achieved by the Emulsification Combined with the Dissolution of Chitosan by $\mathrm{CO}_{2}$ Saturation. Molecules, 24, 4532. https://doi.org/10.3390/molecules24244532

[53] Chinavinijkul, P., Riansuwan, K., Kiratisin, P., Srisang, S. and Nasongkla, N. (2021) Dip- and Spray-Coating of Schanz Pin with PLA and PLA Nanosphere for Prolonged Antibacterial Activity. Journal of Drug Delivery Science and Technology, 65, Article ID: 102667. https://doi.org/10.1016/j.jddst.2021.102667

[54] Botrel, D.A., Fernandes, R.V.D.B. and Borges, S.V. (2015) Microencapsulation of Essential Oils Using Spray Drying Technology. In: Microencapsulation and Microspheres for Food Applications, Elsevier, Amsterdam, 235-251. https://doi.org/10.1016/B978-0-12-800350-3.00013-3 